\title{
SPECTRA OF THEORIES AND STRUCTURES
}

\author{
URI ANDREWS AND JOSEPH S. MILLER
}

\begin{abstract}
We introduce the notion of a degree spectrum of a complete theory to be the set of Turing degrees that contain a copy of some model of the theory. We generate examples showing that not all degree spectra of theories are degree spectra of structures and vice-versa. To this end, we give a new necessary condition on the degree spectrum of a structure, specifically showing that the set of PA degrees and the upward closure of the set of 1-random degrees are not degree spectra of structures but are degree spectra of theories.
\end{abstract}

\section{INTRODUCTION}

The degree spectrum $\operatorname{Spec}(M)$ of a structure $M$ is the set of Turing degrees of presentations of $M$. The collection of spectra of structures has long been studied in computable structure theory. In computable model theory, the main topic is the relationship between the properties of a first order theory and the difficulty of computing presentations or properties of its models. With this viewpoint, we present a new definition. We define the spectrum $\operatorname{Spec}(T)$ of a theory $T$ to be the collection of Turing degrees of presentations of models of $T$. We believe that analyzing spectra of theories will lead to a better understanding of the relationship between the model theoretic properties of a theory and the computability-theoretic complexity of its models.

In Section 2, we show that the class of graphs is universal for theory spectra and show several examples of spectra of theories. In particular, we show that many familiar structure spectra are also theory spectra, including any upward cone, the non-computable degrees, the high degrees, the non-low degrees, the array noncomputable degrees, and the hyperimmune degrees. We also show that some more exotic sets are the degree spectra of theories, including the PA degrees, the upward closure of the set of 1-random degrees, and certain non-degenerate unions of two upward cones of degrees. It is known that no non-degenerate union of two upward cones is the spectrum of a structure, and we show in Section 3 that the PA degrees and the upward closure of the set of 1-random degrees are not structure spectra. We also isolate a property of theory spectra in Lemma 2.16 that allows us to show that some known spectra of structures are not spectra of theories. One example is the non-hyperarithmetical degrees, which were shown to be a structure spectrum by Greenberg, Montalbán and Slaman [10].

In Section 4, we see a connection between theory spectra and a model-theoretic property of the theory. In particular, we show that while the PA degrees, the upward closure of the set of 1-random degrees, and a non-degenerate union of two

Date: September 18, 2014.

2010 Mathematics Subject Classification. 03C57, 03D45.

The second author was supported by the National Science Foundation under grant DMS1001847. 
cones are theory spectra, they are not the spectrum of any atomic theory. This led us to ask if every atomic theory's spectrum is a structure spectrum. We also asked if every $\omega$-stable theory's spectrum is a structure spectrum. The first question was answered by Andrews and Knight [2], who recently showed that the collection of degrees of nonstandard models of true arithmetic is the spectrum of an atomic theory but not a structure spectrum. The second question was recently answered by Andrews, Cai, Diamondstone, Lempp, and Miller [1]. They constructed sets $A$ and $B$ for which the collection of $D$ such that either $A \leq_{T} D$ or $B$ is the range of a $D$-computable limitwise-monotonic function is the spectrum of an $\omega$-stable theory but not the spectrum of any structure. Andrews et al. [1] also showed that the collection of degrees of nonstandard models of true arithmetic is not the spectrum of an $\omega$-stable theory.

Throughout, all theories will be assumed to be complete, and languages will always be assumed to be computable.

\section{Spectra of Theories}

We begin our study of the spectra of theories by paralleling the development of spectra of structures. In both cases, spectra are closed upward, except in the trivial case. A structure $A$ is trivial if there is a tuple of elements $\bar{a}$ such that every permutation of $A$ that fixes $\bar{a}$ pointwise is an automorphism. It is easy to see that the spectrum of a trivial structure consists of a single Turing degree. Knight [13] proved that the spectrum of a non-trivial structure is closed upward.

Proposition 2.1. If a theory $T$ has a trivial model, then $\operatorname{Spec}(T)$ consists of a single degree. Otherwise it is closed upward.

Proof. Note that, by definition, $\operatorname{Spec}(T)=\bigcup_{M \models T} \operatorname{Spec}(M)$.

First, suppose that $T$ has no trivial model. Since Knight's theorem guarantees that $\operatorname{Spec}(M)$ is closed upward for each model $M$ of $T$, so is $\operatorname{Spec}(T)$.

Now, suppose $T$ has a trivial model, and fix $A$ to be such a model of $T$. Let $\bar{a} \in A$ be such that the automorphism group of $A$ acts fully transitively over $\bar{a}$. The number of $n$-types in the language with $\bar{a}$ named is finite, and thus the number of $n$-types in $T$ is finite. Thus $A$ is the unique countable model of $T$ (see [12, Theorem 6.3.1], the Ryll-Nardzewski theorem). So $\operatorname{Spec}(T)=\operatorname{Spec}(A)$, which is a single degree.

Hirschfeldt, Khoussainov, Shore, and Slinko [11] proved that the class of graphs is universal for structure spectra in the sense that every spectrum of a structure is the spectrum of a graph. We prove the analogous result for theory spectra.

Proposition 2.2. We can translate any theory to the language of graphs, preserving the spectrum. This translation preserves atomicity, having a saturated model, stability, superstability, and $\omega$-stability (but does not preserve strong minimality or $\aleph_{0}$-categoricity).

Proof. We may assume that the language of the given theory is relationary with signature $\left\{R_{i} \mid i \in \omega\right\}$ where each $R_{i}$ is $i$-ary. Given a structure $M$, we construct a graph $G_{M}$ whose theory should have the same spectrum as the theory of $M$.

In $G_{M}$, we have three distinguished elements, $a, b$ and $c$. Attached to $a$ is the unique 3-loop in $G_{M}$ (that is, there are two further elements $a^{\prime}, a^{\prime \prime}$ and $E\left(a, a^{\prime}\right) \wedge$ 
$E\left(a^{\prime}, a^{\prime \prime}\right) \wedge E\left(a^{\prime \prime}, a\right)$ holds $)$, attached to $b$ is the unique 5-loop in $G_{M}$, and attached to $c$ is the unique 7-loop in $G_{M}$.

Attached (i.e., edge-connected) to $a$, we create one element $x_{m}$ for each element $m \in M$. We refer to the collection of $x_{m}$ as $X$. For each $i$-tuple $m_{1}, \ldots, m_{i}$ of elements from $M$, we attach an $i+k$-chain to the element $x_{m_{k}}$, so that the last elements of the chains are equal. If $M=R_{i}(\bar{m})$, we attach this last element to $b$, and if $M=\neg R_{i}(\bar{m})$, then we attach this last element to $c$.

We first argue that if $M \equiv N$ then $G_{M} \equiv G_{N}$. Let $T$ be the theory of $M$ and $N$. Take saturated elementary extensions $G_{M} \preccurlyeq A$ and $G_{N} \preccurlyeq B$ of cardinality $\kappa^{1}$. Note that the structures of $X^{A}$ and $X^{B}$ as models of $T$ are definable. So $X^{A}$ and $X^{B}$ are saturated models of $T$ of size $\kappa$. Thus there is an isomorphism taking $X^{A}$ to $X^{B}$, and it is easy to see that this isomorphism extends to every element of the graphs $A$ and $B$ which are connected to $X^{A}$ or $X^{B}$ without going through $b$ or $c$. Each remaining element of $A$ or $B$ comes in one of three configurations: a $\mathbb{Z}$-chain, a collection of $\kappa \omega$-chains emanating from the same element $d$ where $E(d, b)$ holds, and a collection of $\kappa \omega$-chains emanating from the same element $d$ where $E(d, c)$ holds. Saturation implies that there must be $\kappa$ of each of these configurations, so there is a bijection between these configurations in $A$ and $B$ to complete the isomorphism $A \cong B$.

Given a theory $T$, let $T^{\prime}$ be the theory of $G_{M}$ for any $M \models T$. From a dcomputable presentation of any model $G$ of $T^{\prime}$, one can effectively construct a structure whose universe is $X \subseteq G$ and where $R_{i}(\bar{x})$ holds if and only if the final node in the $i+k$-chains connected to each member of $\bar{x}$ is connected to $b$. This gives a d-computable presentation of a structure $M$. Since the relations $R_{i}$, interpreted in this way, are definable in $T^{\prime}$, we see that $T^{\prime}$ includes statements guaranteeing that $M$ models $T$. Similarly, the construction of $G_{M}$ from $M$ gives an effective way to construct models of $T^{\prime}$ from models of $T$. Therefore, $\operatorname{Spec}(T)=\operatorname{Spec}\left(T^{\prime}\right)$. If $A$ is an atomic model of $T$, then $G_{A}$ is an atomic model of $T^{\prime}$, and counting types yields the rest.

We now give several examples of spectra of theories.

Example 2.3. Given any $A \subseteq \omega$, there is a theory $T$ such that $\operatorname{Spec}(T)=\{\mathbf{a} \mid$ $A$ is a-c.e. $\}$.

Proof. Let $M$ be the graph structure comprised of the disjoint union of one $n+3$ cycle for each $n \in A$. Certainly, $M$ is computably presentable from any degree enumerating $A$. Similarly, if a computably presents a model $N \equiv M$, then the set of $n$ for which there exists an $n+3$-cycle in $N$ is $\Sigma_{1}^{0}[\mathbf{a}]$.

Example 2.4. Given any Turing degree $\mathbf{d}$, there is a theory $T$ such that $\operatorname{Spec}(T)$ is the cone above $\mathbf{d}$.

Proof. Fix $D \in \mathbf{d}$. Apply the previous example to $A=D \oplus(\omega \backslash D)$. The degrees a such that $A \in \Sigma_{1}^{0}[\mathbf{a}]$ are exactly the degrees that compute $D$.

For the next example, and the proposition following it, we need to define daisygraphs. An $n$-loop is a connected graph $A$ of size $n$ where every element has valence 2. A daisy is a union of loops ( $n$-loops for various $n$ ) intersecting in exactly one

\footnotetext{
${ }^{1}$ Technically, we are making a set theoretic assumption, but, as usual, this assumption is removable.
} 
element $c$. The element $c$ is called the center of the daisy, and for each loop $A$ of the daisy, we call $A \backslash\{c\}$ a petal. A daisy-graph is a disjoint union of daisies.

Example 2.5. There is a theory $T$ such that $\operatorname{Spec}(T)$ is exactly the non-computable degrees. In fact, for any degree $\mathbf{a}$, the set of all degrees strictly above $\mathbf{a}$ is the spectrum of a theory.

Proof. Wehner [22] constructed a graph $M$ with only finite connected components such that $\operatorname{Spec}(M)$ is exactly the non-computable degrees. The Wehner graph is a daisy-graph representing the sets $n \oplus F$ where $F$ is a finite set that is not equal to $W_{n}$. Let $M^{\prime}$ be the union of countably many copies of $M$. Again, $\operatorname{Spec}\left(M^{\prime}\right)$ is exactly the non-computable degrees. (It is the set of degrees that uniformly enumerate the set $S=\left\{n \oplus F \mid F \neq W_{n}\right\}$ with infinite repetitions. The fact that Wehner's graph is not computable does not depend on the multiplicity of the connected components.) It turns out that there is a computable model of the theory of $M^{\prime}$ (and of $M$ ). For this reason, we extend $M^{\prime}$ to a new structure $N$ such that any model of the theory of $N$ that has a nonstandard copy of $M^{\prime}$ is very complex. Let $N$ be the following structure in signature $\{U(x), E(x, y), R(x, y, z),+(x, y, z), \cdot(x, y, z)\}$ :

- $E(x, y)$ holds only on pairs $(x, y)$ from $U^{N}$.

- $\left(U^{N}, E^{N}\right)$ is isomorphic to $M^{\prime}$.

-,$+ \cdot$ hold only on triples from $(\neg U)^{N}$.

- $\left((\neg U)^{N},+^{N},{ }^{N}\right)$ is isomorphic to $(\mathbb{N},+, \cdot)$.

- $R(x, y, z)$ holds only on triples $(x, y, z)$ where $x, y \in U^{N}$ and $z \in(\neg U)^{N}$.

- For each $E$-connected component $C$ of $U^{N}, R$ is a bijection of $C \times C$ to some initial segment of $(\neg U)^{N}$. (A set $X \subseteq \mathbb{N}$ is an initial segment if it is an initial segment of the linear order $(\mathbb{N},<)$. Since $(\neg U)^{N} \cong(\mathbb{N},+, \cdot)$, we refer to the corresponding sets as initial segments of $(\neg U)^{N}$.)

- For each connected component $C$ of $M$, and each way of bijecting $C \times C$ to an initial segment of $\mathbb{N}$, there are infinitely many copies of $C$ in $U^{N}$ for which $R$ gives this bijection to an initial segment of $(\neg U)^{N}$.

Let $T$ be the theory of $N$. It remains to show that $\operatorname{Spec}(T)$ is precisely the non-computable degrees. From a d-computable presentation of $M^{\prime}$, it is easy to construct a d-computable presentation of $N$. Thus $\operatorname{Spec}(T)$ contains every noncomputable degree. Now let $N^{\prime}=T$. If the $M^{\prime}$ part of $N^{\prime}$ is standard, i.e., if $U^{N^{\prime}}$ is isomorphic to $M^{\prime}$, then $N^{\prime}$ must be non-computable. On the other hand, if the $M^{\prime}$ part of $N^{\prime}$ is nonstandard, then it must contain an infinite $E$-connected component $C$. Fix $x \in C$. It must be the case that $R(x,-,-)$ induces a (partial) injective function from $U^{N^{\prime}}$ to $(\neg U)^{N^{\prime}}$. This function cannot be cofinal, and it must be defined (at least) on all of $C$. Therefore, $(\neg U)^{N^{\prime}}$ must be a nonstandard model of true arithmetic. Feferman [8] proved that a presentation of a nonstandard model of true arithmetic computes every arithmetical set, so once again, $N^{\prime}$ is non-computable.

To prove the relativization, for an arbitrary degree a, take the Wehner graph relative to a and do a similar construction to the one above, but adding a new predicate on the copy of $\mathbb{N}$ to identify $A \subseteq \mathbb{N}$ for some $A \in \mathbf{a}$. The proof is identical, using the fact that a nonstandard model of $\mathbb{N}$ with a predicate for $A$ will compute $A^{(n)}$ for each $n \in \omega$.

Many of the interesting examples of structure spectra given in the literature are either the set of degrees enumerating a family of finite sets, or the set of degrees 
computing a family of finite sets. This includes the previous example, and we can extend the method used there provided that the spectrum includes an arithmetical degree. If $X \subseteq \omega$, we write $X^{[i]}=\{n \mid\langle i, n\rangle \in X\}$ for the $i$ th column of $X$. The columns of $X$ form a family of sets, that is, a collection for which multiplicity matters. We use set notation for families, taking the equality of multiplicities as understood.

Proposition 2.6. If $S$ is a family of finite sets where each set appears infinitely often, then $\left\{\mathbf{d} \mid \exists X \in \Sigma_{1}(\mathbf{d})\left[S=\left\{X^{[i]} \mid i \in \omega\right\}\right]\right\}$ is the spectrum of a theory if it contains an arithmetical degree. Similarly, for any family $S$ of finite sets, $Q:=\left\{\mathbf{d} \mid \exists X \leq_{T} \mathbf{d}\left[S=\left\{X^{[i]} \mid i \in \omega\right\}\right]\right\}$ is the spectrum of a theory if it contains an arithmetical degree.

Proof. For the first claim, we use the construction in Example 2.5. In particular, we define for each $F \in S$ the $F$-daisy comprised of one loop of size $n$ for each $n \in F$. Then we let $W^{\prime}$ be the graph comprised of countably infinitely many $F$-daisies for each $F \in S$. As above, presenting a copy of $W^{\prime}$ is equivalent to being a member of $\left\{\mathbf{d} \mid \exists X \in \Sigma_{1}(\mathbf{d})\left[S=\left\{X^{[i]} \mid i \in \omega\right\}\right]\right\}$. We again want to form a theory whose prime model is computable in $\mathbf{d}$ if and only if $W^{\prime}$ is computable in $\mathbf{d}$, and we want the non-prime models to only be computable from degrees that are upper bounds for all arithmetical degrees. Applying the construction in Example 2.5 to the graph $W^{\prime}$ does exactly this.

To see that the degrees computing a family $S$ of finite sets is the spectrum of a theory, we need to alter that construction slightly. For each $F \in S$, we construct a colored daisy $D_{F}$ that has one blue loop of size $n+3$ for every $n \in F$ and one red loop of size $n+3$ for every $n \notin F$. Let $N$ be the following structure in signature $\{U(x), E(x, y), R(x, y, z),+(x, y, z), \cdot(x, y, z), B(x), R(x)\}:$

- $E(x, y)$ holds only on pairs $(x, y)$ from $U^{N}$.

- $\left(U^{N}, E^{N}\right)$ is isomorphic to $\bigcup_{F \in S} D_{F}$, where $B$ denotes the blue nodes and $R$ denotes the red nodes (note: this union allows as many copies of $D_{F}$ as the number of times $F$ appears in $S$ ).

-,+ hold only on triples from $(\neg U)^{N}$.

- $\left((\neg U)^{N},+^{N}, \cdot^{N}\right)$ is isomorphic to $(\mathbb{N},+, \cdot)$.

- $R(x, y, z)$ holds only on triples $(x, y, z)$ where $x, y \in U^{N}, x$ is the center of a daisy, and $z \in(\neg U)^{N}$.

- For each $D_{F}$ in $U^{N}$ with center $a, R_{a}:=R(a,-,-)$ is a bijection from the blue nodes in $D_{F}$ to an initial segment of $(\neg U)^{N}$.

- If $x$ is on a smaller loop in $D_{F}$ than $y$, then the $R_{a}(x)<R_{a}(y)$. If $x$ and $y$ are neighbors on a petal of $D_{F}$, then $\left|R_{a}(x)-R_{a}(y)\right|=1$ (where $<$ and are understood as given by the isomorphism between $\left((\neg U)^{N},+^{N},{ }^{N}\right)$ and $(\mathbb{N},+, \cdot))$.

It is clear that $N$ is computable from exactly the degrees in $Q$. Suppose that $\mathbf{d}$ computes a model $N^{\prime}$ of $T$. We say that a set $F$ is represented in $N^{\prime}$ if there is a center $a$ of a daisy in $N^{\prime}$ such that $F=\{n \mid$ there is a blue $n+3$-loop attached to $a\}$. It should be clear that every $F \in S$ is represented in $N^{\prime}$ with at least its multiplicity in $S$. If an infinite set $F$ is represented in $N^{\prime}$ at $a$, then $R_{a}$ is an injection of an infinite set into a proper initial segment of $(\neg U)^{N^{\prime}}$. So $(\neg U)^{N^{\prime}}$ must be a nonstandard model of true arithmetic. By Feferman [8], d computes all arithmetical degrees, thus is in $Q$. 
Now assume that all sets represented in $N^{\prime}$ are finite. If the family of represented sets is $S$, then we see that $\mathbf{d}$ is in $Q$. If not, then there is a set represented in $N^{\prime}$ more often than it appears in $S$. Suppose $F$ is this finite set and it appears $k$ times in $S$. Then $T$ includes the statement that if $a_{1}, \ldots, a_{k+1}$ are distinct elements that have blue petals for each $n \in F$, then at least one has some other blue neighbor. Taking $a_{1}, \ldots, a_{k+1}$ to be the centers of the daisies representing $F$ in $N^{\prime}$, the extra blue neighbor cannot be on a finite petal. So there is a blue $\omega$-chain attached to a daisy in $N^{\prime}$ with center $a$. But $T$ includes the statement that all blue neighbors of $a$ are mapped by $R_{a}$ somewhere in $\neg U$, as well as the statement that each blue neighbor of an element mapped somewhere by $R_{a}$ is also mapped somewhere by $R_{a}$. Thus the entire $\omega$-chain is in the domain of $R_{a}$. So $R_{a}$ is an injection of an infinite set into a proper initial segment of $(\neg U)^{N^{\prime}}$. As before, this means that $(\neg U)^{N^{\prime}}$ is a nonstandard model of true arithmetic and $\mathbf{d}$ is in $Q$.

Example 2.7. The hyperimmune degrees, the array non-computable degrees, and the degrees that are not jump traceable are all degree spectra of theories.

Proof. Csima and Kalimullin [3, Corollary 5.3] show that there is a family of finite sets so that the degrees that uniformly compute the family are exactly the hyperimmune degrees. Similarly, Diamondstone, Greenberg and Turetsky [5] construct a family $S$ of finite sets so that exactly the array non-computable degrees compute $S$. They also show that there is a family of finite sets such that the degrees that uniformly enumerate the family (with repetition) are exactly the degrees that are not jump traceable. By Proposition 2.6, each of these collections is the spectrum of a theory.

The next result gives us jump inversion for theory spectra. We refute transfinite jump inversion in Remark 2.19.

Lemma 2.8. Suppose that $\mathcal{S}$ is the spectrum of a theory. Then $\left\{\mathbf{d} \mid \mathbf{d}^{\prime} \in \mathcal{S}\right\}$ is also the spectrum of a theory.

Proof. We may assume that $\mathcal{S}$ is the spectrum of the theory of a graph. We create a new theory $T^{\prime}$ with the spectrum $\left\{\mathbf{d} \mid \mathbf{d}^{\prime} \in \mathcal{S}\right\}$. To define $T^{\prime}$, we use a construction that first appeared in Marker [16]. Given a graph $A$, we define three extensions: $A_{\forall \exists}, A_{\exists \forall}$, and $A_{\forall \exists / \exists \forall}$. Let $A_{\forall \exists}$ be the following structure in signature $\{U(x), V(x), W(x), P(x, y, z), Q(x, y, z), R(x, y, z, w)\}$ :

- $U, V$, and $W$ partition the universe into three infinite sets.

- $P \subseteq U^{2} \times V$ gives a partition of $V$ into infinitely many infinite sets defined by $P_{a, b}=P(a, b,-)$.

- $Q \subseteq U^{2} \times W$ gives a partition of $W$ into infinitely many infinite sets defined by $Q_{a, b}=Q(a, b,-)$.

- $R \subseteq U^{2} \times V \times W$.

- There is an identification of $U$ with $A$ so that the following hold:

- If $A=E(a, b)$, then $R_{a, b}$ defines a bijection between $P_{a, b}$ and $Q_{a, b}$.

- If $A \models \neg E(a, b)$, then there is a single element $u \in P_{a, b}$ so that $R_{a, b}$ defines a bijection between $P_{a, b} \backslash\{u\}$ and $Q_{a, b}$.

We define $A_{\exists \forall}$ to be $B_{\forall \exists}$ where $B$ is the complement graph of $A$. We define $A_{\forall \exists / \exists \forall}$ to be the structure with signature $\left\{U, V_{1}, W_{1}, V_{2}, W_{2}, P_{1}, Q_{1}, R_{1}, P_{2}, Q_{2}, R_{2}\right\}$ so that

- $U, V_{1}, W_{1}, V_{2}, W_{2}$ parition the universe, 
- $\left(U, V_{1}, W_{1}, P_{1}, Q_{1}, R_{1}\right) \cong A_{\forall \exists}$ and $\left(U, V_{2}, W_{2}, P_{2}, Q_{2}, R_{2}\right) \cong A_{\exists \forall}$, and

- $A_{\forall \exists}$ and $A_{\exists \forall}$ use the same identification of $U$ with $A$.

For $T$ the theory of a graph $G$, let $T^{\prime}$ be the theory of $G_{\forall \exists / \exists \forall}$. It is clear that if $A \equiv B$, then $A_{\forall \exists / \exists \forall} \equiv B_{\forall \exists / \exists \forall}$, so this is well defined.

If $B$ is a model of $T^{\prime}$, let

$$
\begin{aligned}
E & :=\left\{(a, b) \mid \forall x \in P_{1, a, b} \exists y \in Q_{1, a, b} R(a, b, x, y)\right\} \\
& =U \backslash\left\{(a, b) \mid \forall x \in P_{2, a, b} \exists y \in Q_{2, a, b} R(a, b, x, y)\right\} .
\end{aligned}
$$

Then $(U, E)$ is a model of $T$. Since $E \in \Delta_{2}(B)$, given any degree $\mathbf{d} \in \operatorname{Spec}\left(T^{\prime}\right)$, we see that $\mathbf{d}^{\prime} \in \operatorname{Spec}(T)$. Conversely, if $\mathbf{d}^{\prime} \in \operatorname{Spec}(T)$, then we have a model $A$ of $T$ for which the edge relation is $\Delta_{2}$ [d]. Using this, $\mathbf{d}$ can build a copy of $A_{\forall \exists / \exists \forall}$, showing that $\mathbf{d} \in \operatorname{Spec}\left(T^{\prime}\right)$.

Using Lemma 2.8, it is easy to see that the high degrees and the non-low degrees are both spectra of theories, as are many other jump classes.

Example 2.9. For all $n, m \in \omega$, both $\left\{\mathbf{d} \mid \mathbf{d}^{(n)} \geq \mathbf{0}^{(m)}\right\}$ and $\left\{\mathbf{d} \mid \mathbf{d}^{(n)}>\mathbf{0}^{(m)}\right\}$ are spectra of theories. Similarly, for any $A \subseteq \omega$ and $k \geq 1$, the set $\left\{\mathbf{d} \mid A \in \Sigma_{k}^{0}[\mathbf{d}]\right\}$ is the spectrum of a theory.

Proof. Apply Lemma 2.8 to the cone above $\mathbf{0}^{(m)}$ and to the set of degrees strictly above $\mathbf{0}^{(m)}$. For the set of all $\mathbf{d}$ such that $A \in \Sigma_{k}^{0}[\mathbf{d}]$, apply Lemma $2.8 k-1$ times to the theory constructed from $A$ in Example 2.3. This gives the set of degrees $\mathbf{d}$ such that $A \in \Sigma_{1}^{0}\left[\mathbf{d}^{(k-1)}\right]$, which is equivalent to $A \in \Sigma_{k}^{0}[\mathbf{d}]$.

The next three examples give us theory spectra that are impossible for structures. In each example, we construct a tree $E \subseteq 2^{<\omega}$ and then use the following construction to code $E$ into a theory $T_{E}$.

Construction 2.10. Given a tree $E$, we generate a theory $T_{E}$ as follows. For each $\sigma \in 2^{<\omega}$, our language has a unary relation symbol $U_{\sigma}$. We also have a binary relation symbol $R$. We describe a structure in this language. Let $U$ denote the elements on which $U_{\lambda}$ holds, where $\lambda$ is the empty string. Let $W$ be the complement of $U$. Then $R \subseteq U \times W$. Every element of $W$ is connected by $R$ to exactly one element of $U$. Every element of $U$ is connected to at most one element of $W$. Let $S$ denote the elements of $U$ connected by $R$ to some element of $W$. Both $S$ and $U \backslash S$ are infinite.

If $\sigma \notin E$, then $U_{\sigma}$ holds on no elements. If $U_{\sigma i}(x)$, where $i \in\{0,1\}$, then $U_{\sigma}(x)$. On the other hand, if $\sigma \in E$ is not terminal and $U_{\sigma}(x)$, then $U_{\sigma i}(x)$ for some $i \in\{0,1\}$. Finally, if $\sigma, \tau \in E$ and $\sigma$ and $\tau$ are incomparable, then the interpretations of $U_{\sigma}$ and $U_{\tau}$ are disjoint. These rules imply that every $x \in U$ is associated to either a terminal node in $E$ or an infinite path in $[E]$. Each terminal node has infinitely many elements of $S$ associated to it, and none of $U \backslash S$. Finally, if $E$ is infinite above $\sigma$, then there are infinitely many elements of both $U \backslash S$ and $S$ on which $U_{\sigma}$ holds.

Everything described so far can be expressed in first-order logic in a straightforward way. To see that this theory is complete, see that a model $M$ of $T$ interprets the structure whose universe is $U(M)$ with the extra symbol $S(x)$ defined by $\exists y R(x, y)$. Further, $T$ is bi-interpretable with its reduct $T^{\prime}$ in this new language. Thus it suffices to show completeness of this $T^{\prime}$. This can be seen by the quantifier 
elimination of $T^{\prime}$. A formula of the form

$$
\varphi(\bar{y}):=\exists x\left(U_{\sigma}(x) \wedge_{i} U_{\tau_{i}}\left(y_{i}\right) \wedge_{i} x \neq y_{i} \wedge \pm S(x) \wedge_{i} \pm S\left(y_{i}\right)\right)
$$

is clearly either equivalent to false if $E$ is finite above $\sigma$ and $\neg S(x)$ is specified, or is equivalent to $\wedge_{i} U_{\tau_{i}}\left(y_{i}\right) \wedge_{i} \pm S\left(y_{i}\right)$ otherwise.

We say that $X$ is an enumeration in $2^{<\omega} \cup 2^{\omega}$ if $X \in 3^{\omega}$ and for each $i$, either $X^{[i]} \in 2^{\omega}$ or there is a $k \in \omega$ so that $\forall j \geq k X^{[i]}(j)=2$ and $\left.X^{[i]}\right|_{k} \in 2^{<\omega}$.

Lemma 2.11. Let $E \subseteq 2^{<\omega}$ be a tree such that the set of terminal nodes in $E$ is computably enumerable. Then the spectrum of the theory $T_{E}$ is exactly the set of degrees that compute an enumeration of a subset of $2^{<\omega} \cup 2^{\omega}$ that is comprised of all terminal nodes in $E$ and a dense set of paths in $[E]$.

Proof. Suppose $\mathbf{d}$ computably presents the model $M \models T_{E}$. Associate to each $a \in M$ the set $\left\{\sigma|M|=U_{\sigma}(a)\right\}$. Using $M$, $\mathbf{d}$ can thus uniformly enumerate these sets. From the collection of $a \in U \backslash S$, this collection includes a dense set of paths through $E$, and the terminal nodes are hit by elements from $S$.

Suppose that $\mathbf{d}$ uniformly enumerates the collection of terminal nodes along with a dense set of paths in $[E]$. Let $X$ be such an enumeration so that either $X^{[i]}$ is a terminal node in $E$ or is a path in $[E]$. Using this $X$, we present a d-computable structure $M_{X}$, the $i^{\text {th }}$ element of which satisfies $U_{\sigma}$ if and only if $\sigma$ is an initial segment of $X^{[i]}$. Let $M_{X}^{\prime}$ be the $\mathbf{d}$ computable structure comprised of countably many disjoint copies of $M_{X}$. Lastly, let $N$ be the structure where $U^{N}$ is isomorphic to $M_{X}^{\prime}$ and an element of $M_{X}^{\prime}$ is $R$-connected to some member of $W^{N}$ if it is in an even numbered copy of $M_{X}$ or is associated with a terminal node. This $N$ is a model of $T_{E}$ and is $\mathbf{d}$-computable.

A function $f: \omega \rightarrow \omega$ is diagonally non-computable $(D N C)$ if $\forall n\left[f(n) \neq \varphi_{n}(n)\right]$, where $\left\{\varphi_{n}\right\}$ is a fixed effective list of all partial computable functions. The $P A$ degrees are the degrees of elements of $\mathrm{DNC}_{2}$, the $\Pi_{1}^{0}$ class of $\{0,1\}$-valued DNC functions. More information about DNC functions and PA degrees can be found, for example, in Downey and Hirschfeldt [6], which is also an excellent reference on 1-random sequences.

Example 2.12. There is a theory $T$ such that $\operatorname{Spec}(T)$ is exactly the PA degrees.

Proof. Take a computable tree $E \subseteq 2^{<\omega}$ such that $[E]=\mathrm{DNC}_{2}$. Let $T$ be the theory $T_{E}$. From a PA degree, one can uniformly compute paths through nonempty $\Pi_{1}^{0}$ classes, hence every PA degree computes an enumeration that includes all terminal nodes in $E$ and a dense set of paths in $[E]$. On the other hand, every path in $[E]$ has PA degree, so by Lemma 2.11, $\operatorname{Spec}(T)$ is exactly the PA degrees.

Example 2.13. There is a theory $T$ such that $\operatorname{Spec}(T)$ is exactly the upward closure of the set of 1-random degrees.

Proof. Let $E \subseteq 2^{<\omega}$ be an infinite computable tree such that every element of $[E]$ is 1-random. For example, $[E]$ could be the complement of the first level of a universal Martin-Löf test. Kučera [14] proved that if $X \in 2^{\omega}$ is 1-random, then every positive measure $\Pi_{1}^{0}$ class contains a tail of $X$. Furthermore, if $[E]$ is nonempty above some $\sigma \in 2^{<\omega}$, then it must have positive measure above $\sigma$, because no 1-random can be contained in a measure zero $\Pi_{1}^{0}$ class. Therefore, the set of tails of $X$ that are contained in $[E]$ is dense in $[E]$. This means that $X$ can compute an enumeration 
that includes all terminal nodes in $E$ and a dense set of paths in $[E]$ by simply listing its tails for as long as each remains on $E$, then for initial segments of tails that leave $E$, taking the leftmost possible extension until a terminal node is found. So by Lemma 2.11, $\operatorname{Spec}\left(T_{E}\right)$ is exactly the upward closure of the set of 1-random degrees.

Example 2.14. There is a theory $T$ such that $\operatorname{Spec}(T)$ is a non-degenerate union of two cones (i.e., the union of two cones where neither cone contains the other).

Proof. Let $A$ and $B$ be incomparable computably enumerable sets. We will construct a theory along the same lines as above, by first defining a tree then coding that tree into a theory. We first construct a computably enumerable tree $E$ such that all paths in $[E]$ compute either $A$ or $B$ and such that each of $A$ and $B$ compute a dense set of paths in $[E]$. Define $E$ as follows. Put $\sigma$ of length $n=\frac{i(i+1)}{2}$ into $E$ at stage $s \geq n$ if for each $k<i, \sigma$ restricted to the interval $\left[\frac{k(k+1)}{2}, \frac{k(k+1)}{2}+k\right]$ is either $0 \frown\left(A_{s} \mid k\right)$ or $1 \frown\left(B_{s} \mid k\right)$.

First note that the set of terminal nodes in $E$ is computably enumerable. In particular, $\sigma$ is a terminal node in $E$ if there is a stage $s$ such that $\sigma \in E_{s}$, both $\sigma \frown 0$ and $\sigma \frown 1$ are not in $E_{s}$, and there is an interval of the form $\left[\frac{k(k+1)}{2}, \frac{k(k+1)}{2}+k\right]$ on which $\sigma$ is defined but is not equal to either $0 \frown\left(A_{s} \mid k\right)$ or $1 \frown\left(B_{s} \mid k\right)$. In other words, $\sigma$ is terminal on $E_{s}$ and either $A$ or $B$ has changed to ensure that $\sigma$ can not be extended after stage $s$.

Now let $\tau$ be a path in $[E]$. Because no initial segment of $\tau$ is terminal, $\tau$ restricted to $\left[\frac{k(k+1)}{2}, \frac{k(k+1)}{2}+k\right]$ must either be $0 \frown(A \mid k)$ or $1 \frown(B \mid k)$. If infinitely many of these intervals start with a 0 , then $\tau$ computes $A$. Otherwise, infinitely many start with a 1 and $\tau$ computes $B$. By Lemma 2.11, any $Z \in \operatorname{Spec}\left(T_{E}\right)$ enumerates a path in $[E]$, hence computes $A$ or $B$.

It remains to show that $A$ and $B$ are in $\operatorname{Spec}\left(T_{E}\right)$. Let $\sigma \in E$ have length $\frac{i(i+1)}{2}$ for some $i$. Using $A$, we can uniformly compute a path on $E$ extending $\sigma$ by appending strings $0 \frown(A \mid k)$ for $k>i$, as long as these extensions continue to be on $E$. If $\sigma$ is extendable to a path in $[E]$, then this process will produce such a path. Otherwise, it will produce a terminal node on $E$. By starting with all $\sigma \in E$, $A$ uniformly enumerates a collection of strings containing all terminal nodes in $E$ and a dense set of paths in $[E]$. By Lemma 2.11, $A \in \operatorname{Spec}\left(T_{E}\right)$. In the same way, $B \in \operatorname{Spec}\left(T_{E}\right)$, so $\operatorname{Spec}\left(T_{E}\right)$ is the union of the two cones above $A$ and $B$.

Corollary 2.15. There is a theory spectrum that is not a structure spectrum.

Proof. Examples 2.12 and 2.13 yield spectra of theories that are not spectra of structures by Theorem 3.9. Similarly, Example 2.14 yields a theory spectrum that is not the spectrum of a structure (see Soskov [21]).

We have seen several examples of spectra of theories, including some spectra that are possible for structures, and some that are not. It is natural to ask if every possible structure spectrum is also a theory spectrum, but it turns out that the two notions are incomparable. To see this, we have to formulate a restriction on the spectra of theories. 
Lemma 2.16. Let $T$ be a theory and assume that $\mathbf{c}$ computes everything computable from both $\mathbf{a}^{(\omega)}$ and $\mathbf{b}^{(\omega)}$. This holds, for example, if $\mathbf{c}=\mathbf{a}^{(\omega)} \wedge \mathbf{b}^{(\omega)}$. If $\mathbf{a}, \mathbf{b} \in$ $\operatorname{Spec}(T)$, then $\mathbf{c} \in \operatorname{Spec}(T)$.

Proof. If $\mathbf{a}, \mathbf{b} \in \operatorname{Spec}(T)$, then $\mathbf{a}^{(\omega)}$ and $\mathbf{b}^{(\omega)}$ both compute $T$. Thus $\mathbf{c}$ computes $T$. By Henkin's construction, c computes (in fact decides) a model of $T$. Thus $\mathbf{c} \in \operatorname{Spec}(T)$.

A routine degree-theoretic result will help us apply Lemma 2.16.

Lemma 2.17. Let $C \geq_{T} D^{\prime}$. Then there are $X$ and $Y$ such that $X^{\prime}, Y^{\prime} \equiv_{T} C$ and $X$ and $Y$ form a minimal pair over $D$.

Proof. Build $X$ and $Y$ by finite initial segments using the oracle $C \equiv_{T} C \oplus D^{\prime}$. Code $D$ into the even positions of $X$ and $Y$. To ensure that $X^{\prime}, Y^{\prime} \equiv_{T} C$, force the jump and code $C$ into the jump exactly as in the proof of the (relativized) Friedberg completeness criterion (for example, see [20, Theorem VI.3.2]). Finally, intersperse the minimal pair requirements. If we have $\sigma \prec X$ and $\tau \prec Y$, search for $\sigma^{\prime} \succ \sigma$ and $\tau^{\prime} \succ \tau$, both consistent with the coding of $D$, and $n$ such that $\varphi_{e}^{\sigma^{\prime}}(n) \neq \varphi_{i}^{\tau^{\prime}}(n)$ (and both converge). If these exists, then extend to $\sigma^{\prime}$ and $\tau^{\prime}$, which ensures that $\varphi_{e}^{X} \neq \varphi_{i}^{Y}$. Otherwise, if $\varphi_{e}^{X}=\varphi_{i}^{Y}$ is total, then $\varphi_{e}^{X} \leq_{T} D$.

Theorem 2.18. There is a structure spectrum that is not a theory spectrum.

Proof. Apply Lemma 2.17 with $D=\emptyset^{(\omega)}$ and $C=\emptyset^{(\omega \cdot 2+2)}$ to get $X$ and $Y$ such that $X^{\prime}, Y^{\prime} \equiv_{T} \emptyset^{(\omega \cdot 2+2)}$ and $X$ and $Y$ form a minimal pair over $\emptyset^{(\omega)}$. There are $A$ and $B$ such that $A^{(\omega)} \equiv_{T} X$ and $B^{(\omega)} \equiv_{T} Y$ (Macintyre [15]).

Goncharov, Harizanov, Knight, McCoy, Miller and Solomon showed that if $\mathcal{S}$ is the spectrum of a structure, then so is $\left\{\mathbf{d} \mid \mathbf{d}^{(\omega+1)} \in \mathcal{S}\right\}$ [9, Lemma 5.5]. Since $\mathcal{S}=\left\{\mathbf{d} \mid \mathbf{d} \geq \mathbf{0}^{(\omega \cdot 2+2)}\right\}$ is a structure spectrum, so is $\left\{\mathbf{d} \mid \mathbf{d}^{(\omega+1)} \geq \mathbf{0}^{(\omega \cdot 2+2)}\right\}$. This gives us a structure whose spectrum contains $A$ and $B$, but not $A^{(\omega)} \wedge B^{(\omega)} \equiv_{T} \emptyset^{(\omega)}$. This cannot be the spectrum of a theory by Lemma 2.16 .

Remark 2.19. Let $\alpha \geq \omega$ be a computable ordinal. Using the argument above, we can refute $\alpha$-jump inversion for theory spectra. In other words, there is a theory spectrum $\mathcal{S}$ such that $\mathcal{S}^{-(\alpha)}=\left\{\mathbf{d} \mid \mathbf{d}^{(\alpha)} \in \mathcal{S}\right\}$ is not a theory spectrum. This stands in contrast to Lemma 2.8, which implies $\alpha$-jump inversion for theory spectra and finite $\alpha$, and to Lemma 5.5 of [9], which gives $\alpha$-jump inversion for structure spectra and computable successor ordinals $\alpha$.

First assume that $\alpha \geq \omega+1$. Let $\mathcal{S}=\left\{\mathbf{d} \mid \mathbf{d} \geq \mathbf{0}^{(\omega+\alpha+1)}\right\}$ and take $A$ and $B$ such that $A^{(\omega+1)} \equiv_{T} B^{(\omega+1)} \equiv_{T} \emptyset^{(\omega+\alpha+1)}$ and $A^{(\omega)} \wedge B^{(\omega)} \equiv_{T} \emptyset^{(\omega)}$. Then by Lemma 2.16, $\mathcal{S}^{-(\alpha)}$ is not a theory spectrum. Note that $\mathcal{S}^{-(\omega+1)}=\left(\mathcal{S}^{-(1)}\right)^{-(\omega)}$, so if $\omega$-jump inversion were possible for theory spectra, then $(\omega+1)$-jump inversion would also be possible. This proves that $\omega$-jump inversion of theory spectra is not possible in general.

Greenberg, Montalbán and Slaman [10] showed that there is a structure whose spectrum is exactly the non-hyperarithmetical degrees. This gives us another example of a structure spectrum that is not a theory spectrum.

Theorem 2.20. The collection of non-hyperarithmetical degrees is not the spectrum of a theory. 
Proof. The proof is similar to the previous theorem. Let $C \geq_{T} \emptyset^{(\omega+1)}$ be nonhyperarithmetical and apply Lemma 2.17 to get $X$ and $Y$ that form a minimal pair over $\emptyset^{(\omega)}$ and such that $X^{\prime}, Y^{\prime} \equiv_{T} C$. In particular, $X$ and $Y$ are nonhyperarithmetical sets. Let $A$ and $B$ be such that $A^{(\omega)} \equiv_{T} X$ and $B^{(\omega)} \equiv_{T} Y$. Then $A$ and $B$ are also non-hyperarithmetical, but $A^{(\omega)} \wedge B^{(\omega)} \equiv_{T} \emptyset^{(\omega)}$. By Lemma 2.16, the collection of non-hyperarithmetical degrees is not the spectrum of a theory.

\section{Spectra of Structures}

In this section we show that the PA degrees and the upward closure of the set of 1-random degrees are not spectra of structures. Our proofs filter through understanding structures with the c.e. extension property, defined below. We show that if $\operatorname{Spec}(M)$ contains the PA degrees, the 1-random degrees, or more generally, the degrees of any nonempty $\Pi_{1}^{0}$ class, then $M$ has the c.e. extension property. We then show that the spectrum of any structure with the c.e. extension property must also contain a degree not computing any member of any special $\Pi_{1}^{0}$ class in $\omega^{\omega}$. Recall that a class is special if it contains no computable member.

Definition 3.1. A structure $M$ has the c.e. extension property (ceep) if every $\exists$ type of a finite tuple in $M$ is c.e. Equivalently, $M$ has the ceep if for every tuple $\bar{a} \in M$, the set of quantifier-free formulas satisfied by finite extensions $\bar{a} \bar{b}$ in $M$ is a c.e. set.

Remark 3.2. In the Russian literature, see for example [7], the c.e. extension property is referred to as local constructivizability.

Remark 3.3. We will be computing from atomic diagrams of structures. To this end, we fix an effective bijection between $\omega$ and the atomic formulas on tuples from $\omega$, the universe of the structure. Then an atomic diagram is viewed as the sequence in $2^{\omega}$ that assigns truth values to each such atomic formula. A finite string decides finitely many truth values, so it gives a quantifier free formulas on a tuple from $\omega$. Fix a distinct tuple $\bar{a}$ from $\mathcal{M}$, i.e., a tuple of distinct elements. We define $E(\bar{a})$ to be the set of strings in $2^{<\omega}$ corresponding to formulas $\sigma(0,1, \ldots, n)$ so that for some distinct tuple $\bar{b}, M=\sigma(\bar{a} \bar{b})$. If $M$ has the ceep, $E(\bar{a})$ is a c.e. set of strings.

Richter $[17,18]$ defined $\mathcal{M}$ to have the recursive extension property if every $\exists$ type is computable. She showed that if $\mathcal{M}$ has the recursive extension property, then $\mathcal{M}$ has a minimal pair of presentations. We extend her result to show that a stronger conclusion follows from, and in fact is equivalent to, the c.e. extension property.

Definition 3.4. The sets $X$ and $Y$ form a $\Sigma_{1}$-minimal pair if $\Sigma_{1}(X) \cap \Sigma_{1}(Y)=\Sigma_{1}$, in other words, if every set computably enumerable in both $X$ and $Y$ is computably enumerable.

Note that $\Sigma_{1}$-minimal pairs are minimal pairs, as every set computable in both $X$ and $Y$ must be both $\Sigma_{1}$ and co- $\Sigma_{1}$, thus computable. Note also that if $X$ is computable, then $X$ and $Y$ always form a $\Sigma_{1}$-minimal pair.

Lemma 3.5. $\mathcal{M}$ has the ceep if and only if $\mathcal{M}$ has a $\Sigma_{1}$-minimal pair of presentations. $^{2}$

\footnotetext{
${ }^{2}$ We thank Antonio Montalbán for suggesting the "only if" direction.
} 
Proof. $(\Leftarrow)$ Every $\exists$-type of a finite tuple in $\mathcal{M}$ is $\Sigma_{1}$ in both presentations. Thus every $\exists$-type of a finite tuple in $\mathcal{M}$ is c.e.

$(\Rightarrow)$ We show that given any set $A$ there is a presentation $B$ of $\mathcal{M}$ that forms a $\Sigma_{1}$-minimal pair with $A$. We build $B$ by building a bijection between $\omega$ and $\mathcal{M}$. At a given stage, we are committed to a map from an initial segment of $\omega$ into $\mathcal{M}$, i.e., a distinct tuple $\bar{a}$ from $\mathcal{M}$. We want to ensure that if $W_{i}^{A}=W_{e}^{B}$, then $W_{e}^{B}$ is c.e. To this aim, we define the set $W=\left\{n \mid \exists \tau \in E(\bar{a})\left[n \in W_{e}^{\tau}\right]\right\}$. If there is an $n \in W \backslash W_{i}^{A}$, then by passing to a distinct $\bar{a}^{\prime}$ that extends $\bar{a}$ and satisfies the formula corresponding to $\tau$, we ensure that $W_{i}^{A} \neq W_{e}^{B}$. If $W \backslash W_{i}^{A}=\emptyset$, then $W_{e}^{B} \subseteq W \subseteq W_{i}^{A}$, so if the two are equal then they must equal the c.e. set $W$.

Interspersing these actions with requirements extending the bijection between $\omega$ and $\mathcal{M}$, we ensure that $B$ is a presentation of $\mathcal{M}$ that forms a $\Sigma_{1}$-minimal pair with $A$. By letting $A$ be any presentation of $\mathcal{M}$ in the beginning, we get a $\Sigma_{1}$-minimal pair of presentations of $\mathcal{M}$.

We now show that if $\operatorname{Spec}(\mathcal{M})$ contains either a nonempty $\Pi_{1}^{0}$ class or a set of degrees of measure 1 , then $\mathcal{M}$ has the ceep. We will show a strong form of the statement that any nonempty $\Pi_{1}^{0}$ class contains a $\Sigma_{1}$-minimal pair.

Proposition 3.6. Let $Y$ be any set and $P$ be a nonempty $\Pi_{1}^{0}$ class. Then there exists $X \in P$ so that $X$ and $Y$ form a $\Sigma_{1}$-minimal pair.

Proof. We may assume that the $\Pi_{1}^{0}$ class $P$ is special. Otherwise, any computable element of $P$ suffices. We force with nonempty $\Pi_{1}^{0}$ classes, starting with $P_{0}=P$. At stage $s$ we have $P_{s}$ and we aim to ensure that if $W_{e}^{X}=W_{i}^{Y}$, then $W_{e}^{X}$ is c.e. Define $W=\left\{n \mid \forall A \in P_{s}\left[n \in W_{e}^{A}\right]\right\}$. It follows from weak König's lemma that $W$ is c.e., and by definition, $W \subseteq W_{e}^{A}$ for any $A \in P_{s}$. If there exists an $n \in W_{i}^{Y} \backslash W$, then we pass to $P_{s+1}=P_{s} \cap\left\{A \mid n \notin W_{e}^{A}\right\}$ and we have ensured that $W_{e}^{X} \neq W_{i}^{Y}$. If there is no such $n$, then $W_{i}^{Y} \subseteq W \subseteq W_{e}^{X}$, so if $W_{e}^{X}=W_{i}^{Y}$, then they both equal the c.e. set $W$. Then $X$ in $\bigcap_{s} P_{s}$ suffices.

Next we show a strong form of the statement that any measure 1 set of degrees contains a $\Sigma_{1}$-minimal pair.

Proposition 3.7. Let $Y$ be any set. Then almost every $X$ forms a $\Sigma_{1}$-minimal pair with $Y$.

Proof. ${ }^{3}$ For any non-c.e. set $A$, the collection of oracles that enumerate $A$ has measure zero (de Leeuw, Moore, Shannon, and Shapiro [4]). Let $C$ be the collection of sets that are $Y$-c.e. but not c.e. Note that $C$ is countable. Therefore, the set of all $X$ for which no element of $C$ is $X$-c.e. (i.e., the $X$ that form a $\Sigma_{1}$-minimal pair with $Y$ ) has measure one.

Now it remains to show that structures with the ceep have presentations that do not compute a member of any special $\Pi_{1}^{0}$ class in $\omega^{\omega}$.

Proposition 3.8. Let $\mathcal{M}$ be a structure with the ceep. Then $\mathcal{M}$ has a presentation that does not compute a member of any special $\Pi_{1}^{0}$ class in $\omega^{\omega}$.

Proof. We will again build the presentation $A$ of $\mathcal{M}$ by building a bijection between $\omega$ and $\mathcal{M}$. As there are only countably many special $\Pi_{1}^{0}$ classes and Turing reductions, it suffices to show how to avoid computing any member of a particular

${ }^{3}$ We thank the referee for suggesting this proof. 
$\Pi_{1}^{0}$ class $P$ in $\omega^{\omega}$ via a particular Turing reduction $\Phi$. Let $P$ be a special $\Pi_{1}^{0}$ class in $\omega^{\omega}$. At stage $s$ when this requirement acts, we are committed to the map from an initial segment of $\omega$ to some tuple $\bar{a}$ in $\mathcal{M}$. We first ask if there is a $\tau$ in $E(\bar{a})$ and an $i \in \omega$ so that $\Phi^{\tau^{\prime}}(i) \uparrow$ for every $\tau^{\prime} \succ \tau$ in $E(\bar{a})$. If such a $\tau$ exists, we pass to a distinct tuple $\bar{a}^{\prime}$ that extends $\bar{a}$ and satisfies the formula corresponding to $\tau$, and we have forced non-totality of $\Phi$. Otherwise, there is a computable sequence $\tau_{1} \preccurlyeq \tau_{2} \preccurlyeq \cdots$ in $E(\bar{a})$ where $\Phi^{\tau_{i}}(i) \downarrow$. As $P$ is a special $\Pi_{1}^{0}$ class, there is an $i$ such that $\Phi^{\tau_{i}}(i)$ has no extension in $P$. We choose a distinct tuple $\bar{a}^{\prime}$ that extends $\bar{a}$ and satisfies the formula corresponding to $\tau_{i}$, ensuring that $\Phi(A) \notin P$. We again intersperse this with requirements extending the bijection between $\omega$ and $\mathcal{M}$.

Putting together Propositions 3.6 and 3.8, we have the following theorem.

Theorem 3.9. Let $P$ be a nonempty $\Pi_{1}^{0}$ class. If $\mathcal{M}$ is a structure that has presentations computable from every element of $P$, then $\mathcal{M}$ has a presentation that does not compute a member of any special $\Pi_{1}^{0}$ class in $\omega^{\omega}$.

The theorem implies that if $P$ is a special $\Pi_{1}^{0}$ class (in Cantor space), then no structure has a spectrum consisting of exactly the upward closure of the degrees of members of $P$. Recall that the elements of the special $\Pi_{1}^{0}$ class $D_{N} C_{2}$ are exactly the PA degrees. Furthermore, the DNC functions form a special $\Pi_{1}^{0}$ class in $\omega^{\omega}$, giving us the following corollary.

Corollary 3.10. The class of PA degrees is not the spectrum of any structure. Furthermore, any structure spectrum containing at least the PA degrees contains a member of non-DNC degree, i.e., one that does not compute a DNC function.

We could similarly apply the theorem to show that the upward closure of the set of 1-random degrees is not the spectrum of a structure, but a stronger result is possible. The second author showed that if the degree spectrum of a structure has measure 1, then it contains a non-DNC degree (unpublished). We show that now as a consequence of the above.

Theorem 3.11 (Miller). If $\mathcal{M}$ has presentations computable from almost every degree, then it has a presentation of non-DNC degree.

Proof. By Propositions 3.7, $\operatorname{Spec}(\mathcal{M})$ contains a $\Sigma_{1}$-minimal pair. Now apply Lemma 3.5 and Proposition 3.8.

This answers Question 5.2 in Shinoda and Slaman [19]. They produced a countable structure with co-meager spectrum such that every presentation computes a 1-generic. They asked if the same is possible with category replaced by measure. By the previous result, if the spectrum of $\mathcal{M}$ has measure one, then there is a presentation of non-DNC degree. Kučera [14] proved that every 1-random computes a DNC function, so such a presentation cannot compute a 1-random.

\section{Spectra of Atomic Theories}

Note that Examples 2.4, 2.5, 2.7, and 2.9 above are constructed using atomic theories, but Examples 2.12, 2.13, and 2.14 are constructed using non-atomic theories. We now show that these last three examples could not have been achieved with atomic theories. This contrasts with the recent example of Andrews and Knight [2], who gave an atomic theory spectrum that is not the spectrum of any structure. 
For the PA degrees and the upward closure of the set of 1-random degrees, we once again use the ceep.

Proposition 4.1. If $T$ is an atomic theory and $\operatorname{Spec}(T)$ contains a $\Sigma_{1}$-minimal pair, then the prime model of $T$ has the ceep.

Proof. Take $A$ and $B$ to be presentations of models of $T$ that form a $\Sigma_{1}$-minimal pair. Any $\exists$-type $p$ that is realized in the prime model of $T$ is also realized in $A$ and in $B$. Thus $p$ is $\Sigma_{1}(A)$ and $\Sigma_{1}(B)$. Therefore, $p$ is c.e.

Combining the results in the last section with the above proposition, we get the following theorem.

Theorem 4.2. Let $P$ be a nonempty $\Pi_{1}^{0}$ class. If $T$ is an atomic theory and $P \subseteq \operatorname{Spec}(T)$, then $\operatorname{Spec}(T)$ contains a degree that does not compute a member of any special $\Pi_{1}^{0}$ class in $\omega^{\omega}$.

Corollary 4.3. Neither the class of PA degrees nor the upward closure of the class of 1-random degrees is the spectrum of any atomic theory. Furthermore, any spectrum of an atomic theory that contains at least the PA degrees or 1-random degrees also contains a member of non-DNC degree.

We also get the analogous statement for spectra containing almost every degree.

Corollary 4.4. If $T$ is an atomic theory and $\operatorname{Spec}(T)$ has measure 1 , then $\operatorname{Spec}(T)$ contains a degree that computes no member of a special $\Pi_{1}^{0}$ class in $\omega^{\omega}$.

Proof. By Proposition 3.7, we know that $\operatorname{Spec}(T)$ contains a $\Sigma_{1}$-minimal pair. Proposition 4.1 tells us that the prime model of $T$ has the ceep. Thus by Proposition 3.8, we see that $\operatorname{Spec}(T)$ contains a degree that computes no member of a special $\Pi_{1}^{0}$ class in $\omega^{\omega}$.

Similarly, Example 2.14 cannot be attained using an atomic theory:

Theorem 4.5. Assume that $\mathbf{a}$ and $\mathbf{b}$ are Turing incomparable and $T$ is an atomic theory. Then $\operatorname{Spec}(T) \neq\{\mathbf{d} \mid \mathbf{a} \leq \mathbf{d}$ or $\mathbf{b} \leq \mathbf{d}\}$.

Proof. Fix sets $A \in \mathbf{a}$ and $B \in \mathbf{b}$. Suppose that $T$ is an atomic theory such that $A, B \in \operatorname{Spec}(T)$. We will construct a presentation $X$ of the prime model $\mathcal{M}$ of $T$ that does not compute $A$ or $B$. We again build the presentation by building a bijection between $\omega$ and $\mathcal{M}$. At a given stage, we are committed to a map from an initial segment of $\omega$ to a distinct tuple $\bar{a}$ from $\mathcal{M}$. We want to ensure that $X$ computes neither $A$ nor $B$. Consider one requirement, say $\Phi^{X} \neq B$. We will use the $A$-ceep property of $\mathcal{M}$ to diagonalize against $X$ computing $B$. As $\operatorname{Spec}(T)$ contains $A$, we see that $\mathcal{M}$ has the ceep relative to $A$, as in Proposition 4.1. We ask whether there exists a $\tau \in E(\bar{a})$ and an $i \in \omega$ so that $\Phi^{\tau}(i) \downarrow \neq B(i)$. If so, we pass to a distinct tuple $\bar{a}^{\prime}$ that extends $\bar{a}$ and satisfies the formula corresponding to $\tau$, and have successfully ensured that $\Phi^{X} \neq B$. If not, then since $E(\bar{a})$ is $A$-c.e. and $B \mathbb{Z}_{T} A$, we know that $\Phi^{X}$ will be partial. So, we need to perform no action to ensure $\Phi^{X} \neq B$. Reversing the roles of $A$ and $B$, requirements of the form $\Phi^{X} \neq A$ are satisfied in the same way. Again, we intersperse such requirements with requirements extending the bijection between $\omega$ and $\mathcal{M}$.

In fact, the same proof works for any non-degenerate union of at least two and at most countably many cones. 


\section{REFERENCES}

[1] Uri Andrews, Mingzhong Cai, David Diamondstone, Steffen Lempp, and Joseph S. Miller. Theory spectra and classes of theories. To appear.

[2] Uri Andrews and Julia F. Knight. Spectra of atomic theories. J. Symbolic Logic, 78(4):11891198, 2013.

[3] Barbara F. Csima and Iskander S. Kalimullin. Degree spectra and immunity properties. Math. Log. Q., 56(1):67-77, 2010.

[4] K. de Leeuw, E. F. Moore, C. E. Shannon, and N. Shapiro. Computability by probabilistic machines. In Automata studies, Annals of mathematics studies, no. 34, pages 183-212. Princeton University Press, Princeton, N. J., 1956.

[5] David Diamondstone, Noam Greenberg, and Daniel Turetsky. Natural large degree spectra. Computability, 2(1):1-8, 2013.

[6] Rodney G. Downey and Denis R. Hirschfeldt. Algorithmic randomness and complexity. Theory and Applications of Computability. Springer, New York, 2010.

[7] Yuri L. Ershov. Definability and computability. Siberian School of Algebra and Logic. Consultants Bureau, New York, 1996.

[8] Solomon Feferman. Arithmetically definable models of formalized arithmetic. Not. Amer. Math. Soc., 5:679, 1958.

[9] Sergey Goncharov, Valentina Harizanov, Julia Knight, Charles McCoy, Russell Miller, and Reed Solomon. Enumerations in computable structure theory. Ann. Pure Appl. Logic, 136(3):219-246, 2005.

[10] Noam Greenberg, Antonio Montalbán, and Theodore Slaman. Relative to any nonhyperarithmetic set. J. Mathematical Logic, 13(01):1250007, 2013.

[11] Denis R. Hirschfeldt, Bakhadyr Khoussainov, Richard A. Shore, and Arkadii M. Slinko. Degree spectra and computable dimensions in algebraic structures. Ann. Pure Appl. Logic, 115(1-3):71-113, 2002.

[12] Wilfrid Hodges. A shorter model theory. Cambridge University Press, Cambridge, 1997.

[13] Julia F. Knight. Degrees coded in jumps of orderings. J. Symbolic Logic, 51(4):1034-1042, 1986.

[14] Antonín Kučera. Measure, $\Pi_{1}^{0}$-classes and complete extensions of PA. In Recursion theory week (Oberwolfach, 1984), volume 1141 of Lecture Notes in Math., pages 245-259. Springer, Berlin, 1985.

[15] John M. Macintyre. Transfinite extensions of Friedberg's completeness criterion. J. Symbolic Logic, 42(1):1-10, 1977.

[16] David Marker. Non $\Sigma_{n}$ axiomatizable almost strongly minimal theories. J. Symbolic Logic, 54(3):921-927, 1989.

[17] Linda Jean Richter. Degrees of unsolvability of models. PhD thesis, 1977. Ph.D. Thesis, University of Illinois at Urbana-Champaign.

[18] Linda Jean Richter. Degrees of structures. J. Symbolic Logic, 46(4):723-731, 1981.

[19] Juichi Shinoda and Theodore A. Slaman. Recursive in a generic real. J. Symbolic Logic, 65(1):164-172, 2000.

[20] Robert I. Soare. Recursively enumerable sets and degrees. Perspectives in Mathematical Logic. Springer-Verlag, Berlin, 1987. A study of computable functions and computably generated sets.

[21] Ivan N. Soskov. Degree spectra and co-spectra of structures. Annuaire Univ. Sofia Fac. Math. Inform., 96:45-68, 2004.

[22] Stephan Wehner. Enumerations, countable structures and Turing degrees. Proc. Amer. Math. Soc., 126(7):2131-2139, 1998.

Department of Mathematics, University of Wisconsin, Madison, Wi 53706-1388, USA

E-mail address: andrews@math.wisc.edu

E-mail address: jmiller@math.wisc.edu 